\title{
Review of: "Membrane interactions and uncoating of Aichi virus, a picornavirus that lacks a VP4"
}

\author{
Francisco Sobrino $^{1}$ \\ 1 Spanish National Research Council
}

Potential competing interests: The author(s) declared that no potential competing interests exist.

This is an interesting and, in general, well conducted piece of work addressing the effect of low pH on the uncoating and membrane interactions of Aichi virus (AiV), a Kobuvirus that can cause gastrointestinal enteric disease in humans, particularly children, livestock and pets. Besides the interest in understanding the biology of Kobuviruses, the topic addressed can be considered as an in interesting model to understand the mechanisms potentially contributing to non-enveloped viral capsids endocytosis and genome release.

While in most picornaviruses the capsid precursor protein VP0 is cleaved into VP4 and VP2 during capsid assembly, Kobuviruses retain an uncleaved VPO. In different picornaviruses VP4 mediates pore formation in the endosomal membrane allowing release of viral RNA into the cytoplasm for replication.

The authors provide evidence that inhibition of endosomal acidification reduces viral multiplication, and that acidic $\mathrm{pH}$ alters the exposure of hydrophobic residues within the capsid, and enhances genome exposure. In addition, synthetic peptide spanning the N-terminus of VPO mediates membrane pore formation in model membranes, an effect analogous to that shown to be exerted by VP4 in other picornaviruses.

Some comments/questions

In other picornaviruses, not all the VPO molecules incorporated into purified virions are reported to be processed to VP4 and VP2.

The use of other inhibitors of endosomal acidification such as concanamycin A that blocks vacuolarATPase, would have improved the characterization of the dependence of Aichi virus on this process. Was the basal virus titer observed at $-1 \mathrm{~h}$ and $0 \mathrm{~h}$ in graphic $1 \mathrm{~B}$ (about $10^{3} \mathrm{PFU}$ ) expected by the authors? The increase in temperature at which Syto9 fluorescence reaches the maximum in Figs $2 \mathrm{~A}\left(55^{\circ} \mathrm{C}\right)$ and $\mathrm{B}$ $\left(60^{\circ} \mathrm{C}\right)$ should be discussed.

The criteria followed to choose the lipid composition of the liposomes used should be commented. Is this composition physiologically related to that of endosomal membranes?

Do the authors have information/hypothesis on how parecho-like viruses permeabilize membranes? In general, this manuscript is well written and it can be followed in an easy manner. Nevertheless, inclusion of a summary of analogies/differences between the results reported here with AiV and those 
reported for other picornavirus would have improved the text and the message. 